\title{
Multiple courses of immunotherapy with different immune cell types for patients with hepatocellular carcinoma after microwave ablation
}

\author{
MING-AN YU ${ }^{1}$, PING LIANG ${ }^{2}$, XIAO-LING YU ${ }^{2}$, ZHI-YU HAN ${ }^{2}$, XUE-JUAN DONG ${ }^{2}$, \\ YU WANG ${ }^{3}, \mathrm{CHAO}_{\mathrm{CHENG}}{ }^{2}$ and $\mathrm{XIN} \mathrm{LI}^{2}$ \\ ${ }^{1}$ Interventional Ultrasound Center, China-Japan Friendship Hospital, Beijing 100029; \\ ${ }^{2}$ Department of Interventional Ultrasound, Chinese PLA General Hospital, Beijing 100853; \\ ${ }^{3}$ Department of Research and Development, Beijing Yongtai Immune Application Technology Co., Ltd., \\ Beijing 101111, P.R. China
}

Received August 26, 2014; Accepted July 9, 2015

DOI: 10.3892/etm.2015.2681

\begin{abstract}
The aim of the present study was to investigate the therapeutic efficacy of immunotherapy after microwave ablation (MWA), which was used to improve liver function, reduce the recurrence rate and enhance survival period in patients with hepatocellular carcinoma (HCC). Between February 2009 and December 2010, 14 patients received immunotherapy after MWA (immunotherapy group) and 15 patients received MWA alone with no post-ablated adjuvant therapy (control group). Immune and liver parameters, recurrence rate and survival time were recorded. The absolute lymphocyte count in the immunotherapy group exceeded that in the control group after 3 courses of immunotherapy $(\mathrm{P}<0.05)$. No significant differences were detected in the lymphocyte subset distribution in the control and immunotherapy patients prior to ablation $(\mathrm{P}>0.05)$; however, certain cytotoxic subsets $\left(\mathrm{CD}^{+} / \mathrm{CD}^{+}, \mathrm{CD} 8^{+} \mathrm{CD} 28^{+}\right.$and $\mathrm{CD}^{+} \mathrm{CD}^{+} 6^{+} \mathrm{CD} 56^{+} \mathrm{T}$ cells) were over-represented and negative regulatory or helper subsets $\left(\mathrm{CD} 4^{+} \mathrm{CD} 8^{+}, \mathrm{CD} 4^{+}, \mathrm{CD} 4^{+} \mathrm{CD} 25^{+}\right)$ were under-represented in the immunotherapy group between 1 and 12 months after immunotherapy $(\mathrm{P}<0.05)$. After 2 courses of immunotherapy the proliferation rate of myeloid dendritic cells and $\mathrm{T}$ lymphocytes, including $\mathrm{CD} 3^{+} / \mathrm{CD}^{+}$lymphocytes, significantly increased $(\mathrm{P}<0.05$ and $\mathrm{P}<0.01$, respectively). In addition, the level of albumin in the immunotherapy group exceeded that in the control group after 3 courses of immunotherapy $(\mathrm{P}<0.05)$. However, the rate of disease-free survival and overall survival within 16 months of MWA did not differ significantly between the two groups $(\mathrm{P}>0.05)$. In conclusion, the results of the present study indicate that immunotherapy
\end{abstract}

Correspondence to: Dr Ping Liang, Department of Interventional Ultrasound, Chinese PLA General Hospital, 28 Fuxing Road, Beijing 100853, P.R. China

E-mail: liangping301@hotmail.com

Key words: hepatocellular carcinoma, immunotherapy, microwave ablation, T-lymphocyte, recurrence improves the immune status and liver function of patients with HCC.

\section{Introduction}

Hepatocellular carcinoma (HCC) is among the most common cancer types worldwide, with a global incidence exceeding 600,000 new cases per year $(1,2)$. Resection and ablation remain the primary treatments for $\mathrm{HCC}$; however, recurrence is common after curative resection or ablation, and is responsible for the majority of patient mortality. Microvascular invasion and micrometastasis are frequently responsible for the post-operative recurrence of $\operatorname{HCC}(3,4)$. Drugs designed to limit HCC typically exhibit intrinsic hepatotoxicity that may further compromise liver function. In addition, HCC cells often express multi-drug resistant proteins, which reduce the efficacy of chemotherapeutics and small molecule drugs for preventing the recurrence of HCC (5).

Due to its favorable safety and efficacy profile, immunotherapy has increasingly been investigated as an innovative adjuvant treatment for the prevention of HCC recurrence. Four randomized controlled trials of HCC immunotherapy following treatments, including resection and transcatheter arterial chemoembolization (TACE) combined with radiofrequency ablation (RFA), have been conducted (6-9). These trials report that immunotherapy was able to increase the rate of recurrence free survival (RFS); however, immunotherapy did not significantly improve overall survival (OS) rates (10).

However, a number of issues concerning HCC immunotherapy remain unclear. The majority of published studies describe an immunotherapy treatment using a single type of immune cell (6-9). The combined effects of different types of successively-administered immune cells, which is more representative of the native immune response to tumor cells, remains to be determined. In addition, the immune cell infusion protocol may potentially be adjusted to complement the specific patient immune status. A dynamic protocol, applied throughout the recovery period, may achieve a more beneficial outcome compared with previously-published fixed protocols 
applied over a relatively short period of time $(6-9,11)$. Finally, studying the precise changes in immune and liver function throughout the therapy may further clarify the impact of immunotherapy.

The present authors have previously reported the outcome of a phase I clinical study of combination therapy with microwave ablation (MWA) and immunotherapy in patients with HCC (12). Three courses of immunotherapy were applied, including immature dendritic cells (DCs), cytokine-induced killer (CIK) cells and cytotoxic T lymphocytes (CTLs), within three months following radical MWA of the HCC. The preliminary conclusion of the study was that this adoptive immunotherapy was safe and successfully ameliorated the loss of peripheral lymphocytes (12). Immature DCs were injected into the marginal area of ablated tumors, myeloid dendritic cells (MDC) were injected into groin lymph nodes, DC-CIK cells and CTLs were injected into the abdominal cavity, and CIK cells were infused intravenously. The results indicated that immunotherapy soon after MWA is a safe procedure that is capable of reducing the viral load in $>50 \%$ of the patients. The percentage of $\mathrm{CD} 4{ }^{+} \mathrm{CD} 25^{+}$ regulatory $\mathrm{T}$ lymphocytes decreased significantly, while the percentage of $\mathrm{CD}^{+}$effector $\mathrm{T}$ cells was increased significantly in the peripheral blood following therapy (12).

In the present study, an adjusted protocol was employed, which involved a prolonged course of immunotherapy and an expanded range of observational indices. The aim of this study was to investigate the influence of immunotherapy on the immune and liver function, rate of tumor recurrence and mortality in patients with HCC.

\section{Patients and methods}

Study design and patients. This single-center, open-label phase II study of MWA followed by adoptive immunotherapy in HCC patients was approved by the Ethics Committee of Chinese PLA General Hospital (Beijing, China).

A total of 29 patients with HCC that were treated at the Department of Interventional Ultrasound in the Chinese PLA General Hospital between February 2009 and December 2010 were enrolled in this study. HCC was diagnosed and staged according to a previously-reported schedule (5). For each patient, a minimum of two contrast-enhanced ultrasound and computed tomography (CT)/magnetic resonance imaging (MRI) images were obtained and analyzed. The inclusion criteria were as follows: i) Single nodular hepatic tumor with a maximum diameter of $5 \mathrm{~cm}$; ii) $\leq 3$ nodular hepatic tumors with a maximum dimension of $3 \mathrm{~cm}$; iii) absence of portal vein thrombosis or extrahepatic metastases; iv) Child-Pugh classification of A or B (13); v) tumor accessible via a percutaneous approach (14); vi) cirrhosis with chronic hepatitis B or $\mathrm{C}$; and vii) $>10 \mathrm{~g} / \mathrm{dl}$ hemoglobin levels, a white blood cell count of $>2 \times 10^{9} / 1$, a platelet count of $>75 \times 10^{9} / 1$, a serum creatinine level of $<110 \mu \mathrm{mol} / 1,<3$ times the upper limit of aspartate aminotransferase (AST), $<2.5$ times the upper limit of serum bilirubin, and a prothrombin time of $<19$ sec. The exclusion criteria were as follows: i) Pregnant or breast-feeding; ii) psychiatric problems, addiction or any other disorder that prevented informed consent; iii) active uncontrolled infection; iv) concurrent systemic corticosteroid treatment; v) systemic autoimmune disease; vi) clinically significant ischemic heart disease or cardiac failure; vii) chemotherapy or radiotherapy within the preceding 6 months; and viii) $<3$ courses of immunotherapy. Written informed consent was obtained from all the subjects.

MVA treatment and grouping. All treatments were performed using sonographic guidance. The instruments, technique and protocol of MWA used in this study are the same as those previously reported (12). Among the 29 eligible patients, 14 were randomly selected to receive immunotherapy following MWA (immunotherapy group) and 15 patients not to receive any post-ablation adjuvant therapy (control group).

Immunotherapy protocol. In order to reduce the liver injury resulting from puncture that was reported in the previous study (12), the protocol was modified as follows: Immunocytes were isolated from $45 \mathrm{ml}$ peripheral blood on the morning of the day of ablation. Tumor tissue was obtained by a sonography-guided biopsy (18G, BARD, Bard International Inc., Covington, GA, USA) using an 18-gauge needle prior to MWA for pathological diagnosis and lysate preparation. After 9 days, the MDCs were resuspended in $1 \mathrm{ml}$ saline and injected into the bilateral groin lymph nodes $(0.5 \mathrm{ml}$ each) under sonographic guidance. After 12 days, DC-CIK cells were resuspended in $25 \mathrm{ml}$ saline and injected into the right abdominal cavity under sonographic guidance. After 15 days CIK cells were resuspended in $50 \mathrm{ml}$ saline and infused intravenously. This protocol was repeated after 1 month, and again after 3 months (Fig. 1). Thereafter, an additional course of immunotherapy was administered after $6,9,12$, 18 and 24 months in the case that the results of blood tests did not meet the following conditions: i) Absolute lymphocyte count, $>1.2 \times 10^{9} / 1$; ii) $\mathrm{CD}^{+} / \mathrm{CD}^{+}, \mathrm{CD}^{+} / \mathrm{CD}^{+}, \mathrm{CD}^{+} / \mathrm{CD} 28^{+}$, $\mathrm{CD}^{+} / \mathrm{CD} 28^{-}$and $\mathrm{CD}^{+} / \mathrm{CD} 16^{+} / \mathrm{CD} 56^{+} \mathrm{T}$ lymphocyte subset counts within the normal range. These subsets were selected as they are associated with the immune state, and influence the prognostic of patients with HCC. The normal ranges were adopted based on our previous survey study on healthy volunteers (15-18).

Preparation of MDCs and effector cells from peripheral blood. Immunocytes were prepared by Beijing Yongtai Immune Application Technology Co., Ltd. (Beijing, China) in a manufacturing practice-compliant facility. MDCs, DC-CIK cells and CIK cells were isolated in accordance with previously reported methods (12). MDCs and DC-CIK cell infusions were guided by ultrasound (Fig. 2). Ultrasound and contrast-enhanced ultrasound were performed using a Sequoia US system (Siemens Healthcare, Mountain View, CA, USA) with a 3.5-5.0-MHz linear multi-frequency transducer. The ultrasound contrast agent used was SonoVue (Bracco Imaging, Milan, Italy).

Clinical outcomes and follow-up. All the patients were diagnosed using histological analysis. Routine blood panel, tumor markers (including $\alpha$-fetoprotein, carcinoembryonic antigen and carbohydrate antigen 19-9), hepatitis B/C viral load and hepatic and renal function, sonography, contrast-enhanced sonography, contrast-enhanced CT and/or contrast-enhanced MRI were performed prior to immunotherapy and at 1, 3, 6, 9, 12, 18 and 24 months after MWA. All CT studies were 
Table I. Baseline clinical characteristics of patients.

\begin{tabular}{|c|c|c|c|}
\hline Characteristic & Control group $(n=15)$ & Immunotherapy group $(n=14)$ & P-value \\
\hline Gender (male/female) & $14 / 1$ & $14 / 0$ & 0.368 \\
\hline Age (years, mean \pm SD) & $54.0 \pm 7.5$ & $51.5 \pm 9.3$ & 0.154 \\
\hline Maximum diameter of tumor $(\mathrm{mm}$, mean $\pm \mathrm{SD})$ & $25.14 \pm 7.87$ & $23.79 \pm 7.30$ & 0.845 \\
\hline Hepatitis B/C & $14 / 1$ & $14 / 0$ & 0.368 \\
\hline Child-Pugh classification (A/B) & $15 / 0$ & $13 / 1$ & 0.261 \\
\hline Pathology (Well/moderately/poorly-differentiated) & $5 / 6 / 4$ & $5 / 7 / 2$ & 0.236 \\
\hline $\operatorname{ALT}(\mu / 1)$ & $41.3 \pm 7.8$ & $42.8 \pm 7.5$ & 0.598 \\
\hline $\operatorname{AST}(\mu / 1)$ & $46.1 \pm 7.2$ & $46.4 \pm 7.9$ & 0.252 \\
\hline Follow-up time (months) & 16 & 19 & 0.663 \\
\hline
\end{tabular}

SD, standard deviation; ALT, alanine aminotransferase; AST, aspartate aminotransferase.

Table II. Absolute T lymphocyte count pre- and post-immunotherapy.

\begin{tabular}{|c|c|c|c|}
\hline T lymphocyte count & Control group $\left(10^{9} / 1 ; n=15\right)$ & Immunotherapy group $\left(10^{9} / 1 ; n=14\right)$ & P-value \\
\hline Pre-immunotherapy & $1.24 \pm 0.85$ & $1.82 \pm 0.86$ & 0.082 \\
\hline Six months post-immunotherapy & $1.36 \pm 0.70$ & $2.10 \pm 0.93$ & 0.038 \\
\hline
\end{tabular}

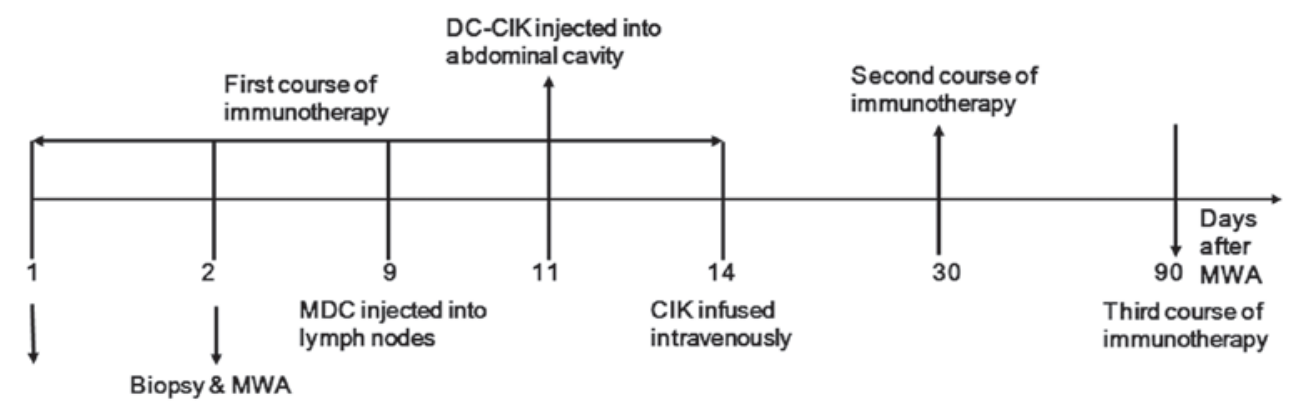

Figure 1. Courses of immunotherapy in patients with hepatocellular carcinoma following MWA. DC-CIK, dendritic cell-cytokine-induced killer; MWA, microwave ablation; MDC, myeloid dendritic cell.

conducted using a multi-detector row CT (Lightspeed 16; GE Medical Systems, Inc., Milwaukee, WI, USA) and contrast medium (iopromide, Ultravist 300; Schering AG, Berlin, Germany). All MRI studies were conducted using a 1.5-T unit (Signa Echo-Speed; GE Medical Systems), and Magnevist contrast medium $(0.1 \mathrm{mmol} / \mathrm{kg}$ body weight; Bayer HealthCare Pharmaceuticals, Leverkusan, Germany).

The disease free survival was defined as the period following immunotherapy when no disease was detectable within 16 months of MWA.

Statistical analysis. Data was analyzed using SAS statistical software, version 8.2 for Windows (SAS Institute Inc., Cary, NC, USA). The continuous data were expressed as mean \pm standard deviation. Multiple groups were compared using analysis of variance or rank sum test. Paired groups were compared by independent-samples t-test, rank sum test or $\chi^{2}$ test. Patient survival time was compared between groups using a Kaplan-Meier survival curve and log-rank tests.
Two-tailed $\mathrm{P}<0.05$ was considered to indicate a statistically significant difference.

\section{Results}

Baseline characteristics of patients. A total of 29 patients with $\mathrm{HCC}$ were recruited in the present study from the Department of Interventional Ultrasound in the Chinese PLA General Hospital between February 2009 and December 2010. A group of 14 patients opted to receive immunotherapy after MWA (immunotherapy group), and 15 patients opted not to receive any post-ablation adjuvant therapy (control group). The baseline characteristics of patients in the two groups did not differ significantly ( $P>0.05$; Table I). By December 2010, 64 courses of immunotherapy had been performed in 14 patients, including 3 courses of immunotherapy completed in 6 patients, 4 courses in 2 patients, 5 courses in 1 patient, 6 courses in 3 patients, 7 courses in 1 patient and 8 courses in 1 patient. One patient experienced severe abdominal pain $30 \mathrm{~min}$ after 
Table III. Comparison of T-lymphocyte subgroup distribution prior to and following immunotherapy.

\begin{tabular}{|c|c|c|c|c|c|c|}
\hline \multirow[b]{2}{*}{ Lymphocyte subset } & \multirow[b]{2}{*}{ Pre-immunotherapy } & \multicolumn{5}{|c|}{ Time post-immunotherapy (months) } \\
\hline & & 1 & 3 & 6 & 9 & 12 \\
\hline $\mathrm{CD}^{+}$ & 0.711 & $0.000^{\mathrm{a}}$ & 0.371 & 0.432 & 0.436 & 0.462 \\
\hline $\mathrm{CD}^{+}$ & 0.843 & 0.115 & $0.026^{\mathrm{a}}$ & 0.092 & 0.115 & 0.774 \\
\hline $\mathrm{CD}^{+}{ }^{+} \mathrm{CD} 28^{+}$ & 0.323 & $0.004^{\mathrm{a}}$ & $0.036^{\mathrm{a}}$ & 0.873 & $0.022^{\mathrm{a}}$ & 0.607 \\
\hline NKT & 0.793 & $0.038^{\mathrm{a}}$ & 0.771 & 0.160 & $0.038^{\mathrm{a}}$ & $0.004^{\mathrm{a}}$ \\
\hline $\mathrm{CD}^{+} \mathrm{CD} 28^{-}$ & 0.585 & 0.432 & 0.777 & 0.326 & 0.471 & 0.948 \\
\hline $\mathrm{CD} 4^{+} \mathrm{CD} 25^{+}$ & 0.915 & 0.544 & $0.024^{\mathrm{b}}$ & $0.025^{\mathrm{b}}$ & 0.795 & 0.629 \\
\hline $\mathrm{CD}^{+}{ }^{+} \mathrm{CD}^{+}$ & 0.743 & 0.585 & $0.008^{\mathrm{b}}$ & 0.065 & 0.359 & 0.879 \\
\hline $\mathrm{CD}^{+}$ & 0.674 & 0.053 & $0.027^{\mathrm{b}}$ & 0.278 & 0.480 & 0.311 \\
\hline $\mathrm{CD}^{+}{ }^{+} \mathrm{CD} 16^{+} \mathrm{CD} 6^{+}(\mathrm{NK})$ & 0.647 & 0.201 & 0.694 & 0.875 & 0.750 & 0.642 \\
\hline $\mathrm{CD}{ }^{+}$ & 0.763 & 0.057 & 0.817 & 0.529 & 0.289 & 0.706 \\
\hline
\end{tabular}

${ }^{\mathrm{a}} \mathrm{T}$ lymphocyte subgroup is higher in patients receiving immunotherapy compared with the control group; ${ }^{\mathrm{b}} \mathrm{T}$ lymphocyte subgroup is lower in patients receiving immunotherapy group compared with the control group. NK, natural killer.

Table IV. Comparison of liver function between immunotherapy and control groups.

\begin{tabular}{lcc}
\hline Serum parameter & Control group $(\mathrm{n}=15)$ & Immunotherapy group $(\mathrm{n}=14)$ \\
\hline ALT $(\mu / \mathrm{l})$ & & $42.8 \pm 7.5$ \\
Pre-immunotherapy & $41.3 \pm 7.8$ & $37.9 \pm 6.2$ \\
Post-immunotherapy & $37.5 \pm 6.3$ & $-4.9 \pm 5.2$ \\
D-value of pre and post-immunotherapy & $-3.7 \pm 7.2$ & 0.598 \\
AST $(\mu / \mathrm{l})$ & & $46.4 \pm 7.9$ \\
Pre-immunotherapy & $46.1 \pm 7.2$ & $37.7 \pm 5.3$ \\
Post-immunotherapy & $35.8 \pm 6.5$ & $-8.7 \pm 5.6$ \\
D-value of pre and post-immunotherapy & $-10.7 \pm 8.8$ & 0.632 \\
Albumin (g/l) & & $41.31 \pm 3.24$ \\
Pre-immunotherapy & $39.27 \pm 4.89$ & $44.39 \pm 3.87$ \\
Post-immunotherapy & $38.43 \pm 5.98$ & $3.07 \pm 2.29$ \\
D-value of pre and post-immunotherapy & $-0.84 \pm 4.63$ & 0.268 \\
ChE $(\mu / 1)$ & & 0.556 \\
Pre-immunotherapy & $5503.49 \pm 1246.45$ & 0.200 \\
Post-immunotherapy & $5701.98 \pm 1303.10$ & 0.004 \\
D-value of pre and post-immunotherapy & $198.49 \pm 562.76$ & 0.008 \\
\hline
\end{tabular}

D-value is the difference between the liver function index pre- and post-immunotherapy. ALT, alanine aminotransferase; AST, aspartate aminotransferase; $\mathrm{ChE}$, cholinesterase.

the sixth DC-CIK infusion into the right abdominal cavity; however, the symptom disappeared rapidly with anti-allergy treatment. No grade III/IV severe adverse events occurred as a result of the other 63 courses of immunotherapy $(19,20)$. However, a fever of $<39^{\circ} \mathrm{C}$ occurred in 28 courses $(43.1 \%)$, in 6 patients $(42.9 \%)$, but all fevers were resolved within $4-24 \mathrm{~h}$ without intervention.

Immunotherapy improves the immunostatus and liver function of patients with HCC. The absolute lymphocyte counts of patients in the control and immunotherapy groups did not differ significantly prior to ablation $(\mathrm{P}>0.05)$. However, after 6 months and following 3 courses of immunotherapy, the mean absolute lymphocyte count in the immunotherapy group $\left(2.10 \pm 0.93 .10^{9} / 1\right)$ significantly exceeded that in the control group $\left(1.36 \pm 0.70 .10^{9} / 1 ; \mathrm{P}<0.05\right.$; Table II).

The lymphocyte subset distribution of patients in the control and immunotherapy groups did not differ significantly prior to ablation $(\mathrm{P}>0.05)$; however, certain cytotoxic subsets $\left(\mathrm{CD}^{+} / \mathrm{CD}^{+}, \mathrm{CD}^{+} \mathrm{CD} 28^{+}\right.$and $\left.\mathrm{CD}^{+} \mathrm{CD} 16^{+} \mathrm{CD} 56^{+}\right)$were over-represented and negative regulatory or helper subsets $\left(\mathrm{CD} 4^{+} \mathrm{CD} 8^{+}, \mathrm{CD}^{+}\right.$and $\left.\mathrm{CD} 4^{+} \mathrm{CD} 25^{+}\right)$were under-represented 
Table V. T cell count and different subgroup ratio after culture between third and first immunotherapy.

\begin{tabular}{|c|c|c|c|}
\hline Parameter & After first immunotherapy $(n=14)$ & After third immunotherapy $(n=14)$ & P-value \\
\hline \multicolumn{4}{|c|}{ Cell count pre-culture } \\
\hline $\operatorname{PBMC~(x10^{7}/1)}$ & $8.93 \pm 4.07$ & $7.32 \pm 2.96$ & 0.996 \\
\hline \multicolumn{4}{|c|}{ Cell count post-culture } \\
\hline $\operatorname{MDC}\left(\times 10^{7} / 1\right)$ & $10.74 \pm 5.26$ & $16.5 \pm 9.16$ & 0.052 \\
\hline CIK $\left(x 10^{10} / 1\right)$ & $2.65 \pm 2.29$ & $3.23 \pm 1.46$ & 0.424 \\
\hline \multicolumn{4}{|c|}{ T cell subgroup ratio post-culture $(\%)$} \\
\hline $\mathrm{CD}_{83} 3^{+}$ & $50.60 \pm 28.65$ & $74.87 \pm 20.87$ & 0.004 \\
\hline $\mathrm{CD}^{+} 6^{+}$ & $62.69 \pm 29.50$ & $86.71 \pm 12.77$ & 0.011 \\
\hline $\mathrm{CD}^{+} \mathrm{CD}^{+}$ & $68.70 \pm 15.73$ & $81.80 \pm 9.26$ & 0.000 \\
\hline $\mathrm{CD}^{+}{ }^{+} \mathrm{CD} 56^{+}$ & $29.59 \pm 11.42$ & $35.82 \pm 8.92$ & 0.058 \\
\hline
\end{tabular}

PBMC, peripheral blood mononuclear cell; MDC, myeloid dendritic cell; CIK, cytokine-induced killer.
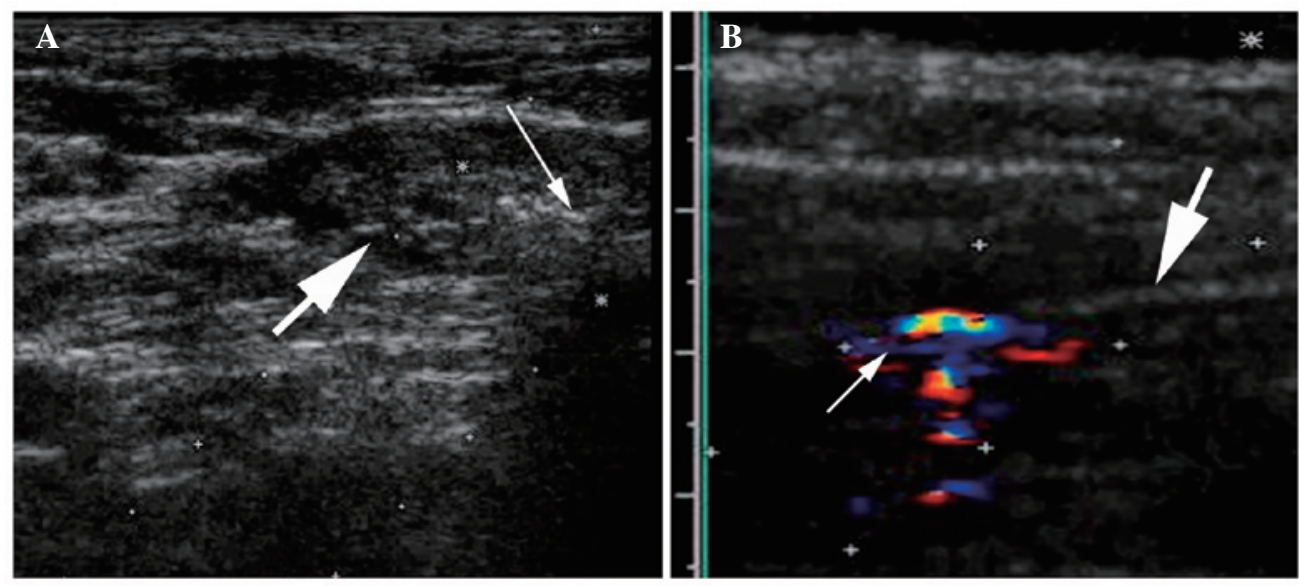

Figure 2. (A) Myeloid dendritic cells (MDCs) injected into groin lymph nodes under sonographic guidance. Local slightly hyperechoic (thin arrow) indicates the MDCs injected into the lymphocyte node (thick arrow). (B) Dendritic cell-cytokine-induced killer (DC-CIK) cells injected into abdominal cavity under sonographic guidance. Color Doppler (thin arrow) shows the process of DC-CIK cells injected into the abdominal cavity (thick arrow, parietal peritoneum).

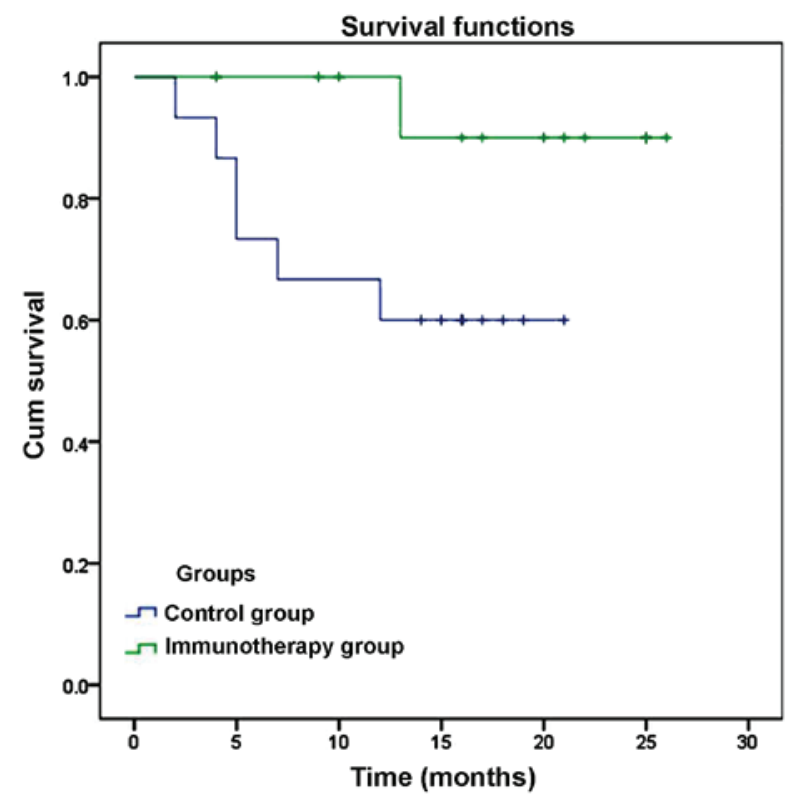

Figure 3. Comparison of disease free survival rates of patients with hepatocellular carcinoma between the immunotherapy and control groups. in the immunotherapy group between 1 and 12 months after immunotherapy $(\mathrm{P}<0.05$; Table III).

The alanine aminotransferase (ALT), AST, albumin and cholinesterase (ChE) levels of patients in the control and immunotherapy groups did not differ significantly prior to ablation. However, after 6 months and 3 courses of immunotherapy, the mean albumin level in the immunotherapy group $(44.39 \pm 3.87 \mathrm{~g} / 1)$ exceeded that in the control group $(38.43 \pm 5.98 \mathrm{~g} / 1 ; \mathrm{P}<0.05$; Table IV). By contrast, the mean ALT, AST and ChE levels did not differ significantly between the control and immunotherapy groups after 6 months (Table IV).

Immunotherapy increased the proliferation of immune cells. The proliferative activity of immune cells was compared after 1 and 3 courses of immunotherapy (Table V). The peripheral blood mononuclear cell (PBMC) count after the first course of immunotherapy $\left(8.93 \pm 4.07 \times 10^{7}\right.$ cells $\left./ 1\right)$ did not differ significantly from the PBMC count after the third course of immunotherapy $\left(7.32 \pm 2.96 \times 10^{7}\right.$ cells $\left./ 1 ; P=0.996\right)$. Furthermore, following in vitro culture, the absolute number of MDCs and CIK cells did not differ significantly between samples collected 
after the first $(10.74 \pm 5.26$ and $2.65 \pm 2.29$, respectively) or third course of immunotherapy $(16.5 \pm 9.16$ and $3.23 \pm 1.46$, respectively). However, the fraction of $\mathrm{CD}^{+}, \mathrm{CD}^{+} 6^{+}$and $\mathrm{CD}^{+} \mathrm{CD}^{+}$cells was significantly increased between the first $(50.60 \pm 28.65,62.69 \pm 29.50$ and $68.70 \pm 15.73$, respectively) and the third courses of immunotherapy $(74.87 \pm 20.87,86.71 \pm 12.77$

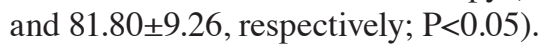

Rate of recurrence and disease-free survival (DFS). Intrahepatic recurrence was detected in 1 case (7.1\%) at 13 months after ablation, but no extrahepatic recurrences or fatalities were detected in the immunotherapy group. In the control group, intrahepatic recurrence was identified in 5 patients $(33.3 \%)$ within 1-19 months of ablation, and extrahepatic recurrence occurred in 1 patient $(6.7 \%)$. In addition, 3 patients in the control group succumbed to various causes at 7, 10 and 12 months after ablation $(20 \%)$. The cause of mortality was upper gastrointestinal bleeding in 1 case, liver failure in 1 case and tumor progression in 1 case. However, due to the low rate of recurrence and mortality, the rate of DFS and overall survival (OS) was not significantly lower in the immunotherapy group compared with the control group (DFS, 92.86 vs. $66.67 \%, \mathrm{P}=0.076$; OS, $100 \%$ vs. $80 \%, \mathrm{P}=0.126$ ), as indicated by a Kaplan-Meier plot (Fig. 3).

\section{Discussion}

Immunotherapy has been demonstrated to increase the rate of recurrence-free survival following resection or ablation in patients with HCC $(6-9,11)$. The aim of the present study was to assess the efficacy of an expanded immunotherapy protocol and to evaluate the precise differences in immune and liver function in order to clarify the impact of immunotherapy. In a previous study, the present authors reported the outcome of a phase I clinical study of immunotherapy, which included immature DCs, CIK cells and CTL following MWA of HCC (12). In the present study, a prolonged course of immunotherapy was administered to patients and an expanded range of observational indices were detected.

The absolute lymphocyte count in the immunotherapy group exceeded that in the control group after 3 courses of immunotherapy. Furthermore, certain cytotoxic subsets $\left(\mathrm{CD}^{+} / \mathrm{CD}^{+}\right.$, $\mathrm{CD}^{+} \mathrm{CD} 28^{+}$and $\mathrm{CD}^{+} \mathrm{CD}^{+} 6^{+} \mathrm{CD}^{2} 6^{+}$) were over-represented, while negative regulatory or helper subsets $\left(\mathrm{CD} 4^{+} \mathrm{CD} 8^{+}, \mathrm{CD} 4^{+}\right.$ and $\mathrm{CD}^{+} \mathrm{CD}_{25} 5^{+}$were under-represented in the immunotherapy group between 1 and 12 months after immunotherapy. After 2 courses of immunotherapy the proliferation rate of MDCs and $\mathrm{T}$ lymphocytes, including $\mathrm{CD}^{+} / \mathrm{CD}^{+}$cells, was significantly increased. In addition, the level of albumin in the immunotherapy group was increased compared with the control group after 3 courses of immunotherapy, indicating the accelerated recovery of liver function. However, the rate of disease free survival and overall survival within 16 months of MWA did not differ significantly between the two groups.

The present results indicate that the immunotherapy protocol induced a wide spectrum of changes in the immune system. A number of studies have previously reported that an elevated ratio of lymphocyte to monocytes pre- or postoperatively is positively correlated with the outcomes of patients with various malignancies $(21,22)$. Increased lymphocyte counts have been observed following immunotherapy, indicating elevated antitumor activity (23). The proliferative capacity of patient T lymphocytes was elevated after 2 courses of immunotherapy. The $\mathrm{MDC}, \mathrm{CD}^{+}$and $\mathrm{CD}^{+} \mathrm{CD}^{2} 6^{+} \mathrm{CD} 56^{+} \mathrm{T}$ cell subsets appeared to be particularly enhanced $(24,25)$. Enhanced levels of cytotoxic subsets $\left(\mathrm{CD}^{+} / \mathrm{CD}^{+}, \mathrm{CD} 8^{+} \mathrm{CD} 28^{+}\right.$and $\left.\mathrm{CD}^{+} \mathrm{CD}^{+} 6^{+} \mathrm{CD}^{2} 6^{+}\right)$were detected in the peripheral blood, within 12 months of treatment, indicating an enhanced antitumor immune response $(26,27)$. Immune regulatory or suppressive subsets have been reported to be elevated in the peripheral blood at 3-6 months after ablation, relieving the immune response directed towards HCC (28). For the suppressive subset $\mathrm{CD} 4{ }^{+} \mathrm{CD} 25^{+}$, the levels were reduced between 3 and 6 months after ablation, which partly mitigated the immunosuppressive response to HCC (28). In addition, reduced levels of the suppressive and immunoregulatory subsets $\left(\mathrm{CD} 4^{+} \mathrm{CD}^{+}, \mathrm{CD}^{+}\right.$and $\left.\mathrm{CD} 4^{+} \mathrm{CD} 25^{+}\right)$were observed between 1 and 12 months after MWA. However, this difference may be attributed to the increase in the ratio in cytotoxic subsets.

In the current study, immunotherapy appeared to accelerate the recovery of circulating albumin levels, which is an indicator of liver function. However, no statistically significant differences were detected in other measures of liver function (including ChE, AST and ALT) between patients that received immunotherapy and those in the control group. The observed recovery of albumin levels in the present study demonstrates the improvement of liver cell status and indicates the improvement of the liver microenvironment.

The disease free survival and overall survival rate were not significantly higher in the immunotherapy group compared with the control group. However, it is possible that a larger study in which more patients are enrolled and followed-up for a longer time period ( $>19$ months) may reveal the impact of immunotherapy of outcomes to be significant. Previous studies including longer follow-up periods (up to 5 years post-ablation) $(6,7)$, or enrolling larger numbers of patients (6-8) have reported an impressive impact of immunotherapy on recurrence rates and outcome. Therefore, future studies involving more patients for a longer follow-up period are required. Furthermore, studies should investigate the cytotoxic capacity of immune cells ex vivo, in order to determine whether immunotherapy may exert the observed beneficial effects by enhancing the capacity of immune cells to destroy remaining or recurring $\mathrm{HCC}$ cells.

In conclusion, in the present study, an immunotherapy protocol employing multiple types of immune cells, administered after MWA, was found to improve the immune status and liver function in patients with HCC.

\section{Acknowledgements}

The study was supported by grants from the National Scientific Foundation Committee of China (no. 81127006), Science Technology Support (no. 2013BAI01B01) and International Cooperation Project (no. 2012DFG32070).

\section{References}

1. El-Serag HB: Hepatocellular carcinoma: recent trends in the United States. Gastroenterology 127 (5 Suppl 1): 27-34, 2004.

2. Bosetti C, Levi F, Boffetta P, Lucchini F, Negri E and La Vecchia C: Trends in mortality from hepatocellular carcinoma in Europe, 1980-2004. Hepatology 48: 137-145, 2008. 
3. Kaibori M, Ishizaki M, Matsui K and Kwon AH: Predictors of microvascular invasion before hepatectomy for hepatocellular carcinoma. J Surg Oncol 102: 462-468, 2010.

4. Funaki NO, Tanaka J, Seto SI, Kasamatsu T, Kaido T and Imamura M: Hematogenous spreading of hepatocellular carcinoma cells: Possible participation in recurrence in the liver. Hepatology 25: 564-568, 1997.

5. Bruix J, Sherman M, Llovet JM, et al: Clinical management of hepatocellular carcinoma. Conclusions of the Barcelona-2000 EASL conference. European association for the study of the liver. J Hepatol 35: 421-430, 2001.

6. Takayama T, Sekine T, Makuuchi M, et al: Adoptive immunotherapy to lower postsurgical recurrence rates of hepatocellular carcinoma: A randomised trial. Lancet 356: 802-807, 2000.

7. Hui D, Qiang L, Jian W, Ti Z and Da-Lu K: A randomized, controlled trial of postoperative adjuvant cytokine-induced killer cells immunotherapy after radical resection of hepatocellular carcinoma. Dig Liver Dis 41: 36-41, 2009.

8. Weng DS, Zhou J, Zhou QM, et al: Minimally invasive treatment combined with cytokine-induced killer cells therapy lower the short-term recurrence rates of hepatocellular carcinomas. J Immunother 31: 63-71, 2008.

9. Zhou WP, Wu MC and Chen H: The effects of combined hepatectomy and immuno-chemotherapy on postoperative recurrence rate of primary liver cancer. Zhong Hua Wai Ke Za Zhi 33: 35-37, 1995 (In Chinese).

10. Zhong JH, Ma L, Wu LC, et al: Adoptive immunotherapy for postoperative hepatocellular carcinoma: A systematic review. Int J Clin Pract 66: 21-27, 2012.

11. Ma H, Zhang Y, Wang Q, et al: Therapeutic safety and effects of adjuvant autologous RetroNectin activated killer cell immunotherapy for patients with primary hepatocellular carcinoma after radiofrequency ablation. Cancer Biol Ther 9: 903-907, 2010.

12. Zhou P, Liang P, Dong B, Yu X, Han Z and Xu Y: Phase clinical study of combination therapy with microwave ablation and cellular immunotherapy in hepatocellular carcinoma. Cancer Biol Ther 11: 450-456, 2011.

13. Zhang J, Ye L, Zhang J, Lin M, He S, Mao X, Zhou X and Zhi F: MELD scores and Child-Pugh classifications predict the outcomes of ERCP in cirrhotic patients with choledocholithiasis: A retrospective cohort study. Medicine (Baltimore) 94: e433, 2015.

14. Liu SR, Liang P, Yu XL, Cheng ZG, Han ZY and Yu J: Percutaneous microwave ablation for liver tumours adjacent to the marginal angle. Int J Hyperthermia 30: 306-311, 2014.

15. Usuda S, Yoshizawa K, Yabu K and Kiyosawa K: Immunological responses against an autologous human hepatocellular carcinoma cell line. J Gastroenterol Hepatol 8: 517-523, 1993.

16. Kong L, Yao SK, Liu JX and Wang N: The prognostic value of cellular immunity function in patients with hepatocellular carcinoma. Zhonghua Gan Zang Bing Za Zhi 13: 194-197, 2005.
17. Deguchi Y, Yoshimatsu K and Endo S: Natural killer-like T cell lymphoma of the small intestine: Report of a case. Surg Today 36: 474-477, 2006.

18. Liu HR and Li WM: Treg-specific demethylated region activity in isolated regulatory t lymphocytes is a surrogate for disease severity in hepatocellular carcinoma. IUBMB Life 67: 355-360, 2015.

19. Passalacqua G, Baena-Cagnani CE, Bousquet J, Canonica GW, Casale TB, Cox L, Durham SR, Larenas-Linnemann D, Ledford D, Pawankar R, et al: Grading local side effects of sublingual immunotherapy for respiratory allergy: Speaking the same language. J Allergy Clin Immunol 132: 93-98, 2013.

20. Cox L, Larenas-Linnemann D, Lockey RF and Passalacqua G: Speaking the same language: The World Allergy Organization subcutaneous immunotherapy systemic reaction grading system. J Allergy Clin Immunol 25: 569-574, 2010.

21. Song R, Ikeguchi M, Zhou G and Kuo MT: Identification and characterization of a hepatoma cell-specific enhancer in the mouse multidrug resistance mdrlb promoter. J Biol Chem 270 25468-25474, 1995.

22. Chen TM, Lin CC, Huang PT and Wen CF: Neutrophil-to-lymphocyte ratio associated with mortality in early hepatocellular carcinoma patients after radiofrequency ablation. J Gastroenterol Hepatol 27: 553-561, 2012.

23. Koh YW, Kang HJ, Park C, et al: The ratio of the absolute lymphocyte count to the absolute monocyte count is associated with prognosis in Hodgkin's lymphoma: correlation with tumor-associated macrophages. Oncologist 17: 871-880, 2012.

24. Lee CS: Lymphocytes and their subsets in the peripheral blood of hepatocellular carcinoma patients. J Formos Med Assoc 90: 626-630, 1991

25. Wang JX, Liu GH, Fan YZ, et al: Effects of cytotoxic T lymphocytes on hepatoma cell line SMMC-7721 induced by different subsets of dendritic cells in vitro. Hepatobiliary Pancreat Dis Int 5: 422-427, 2006.

26. Olioso P, Giancola R, Di Riti M, Contento A, Accorsi P and Iacone A: Immunotherapy with cytokine induced killer cells in solid and hematopoietic tumours: A pilot clinical trial. Hematol Oncol 27: 130-139, 2009.

27. Qiu YR, Yang CL, Chen LB and Wang Q: Analysis of CD8 (+) and CD8 (+) CD28 (-) cell subsets in patients with hepatocellular carcinoma. Di Yi Jun Yi Da Xue Xue Bao 22: 72-73, 2002 (In Chinese).

28. Shibolet O, Alper R, Zlotogarov L, et al: Suppression of hepatocellular carcinoma growth via oral immune regulation towards tumor-associated antigens is associated with increased NKT and CD8+lymphocytes. Oncology 66: 323-330, 2004. 